\title{
Expression of Cyclin D1 in Hyperplasia and Carcinoma of Endometrium and Its Correlation with Histologic Grade, Tumor Type, and Clinicopathological Features
}

\author{
Karuna Sangwan ${ }^{1}$ Monika Garg² Nayana Pathak ${ }^{3}$ \\ ${ }^{1}$ Department of Pathology, World College of Medical Science and \\ Research, Jhajjar, Haryana, India \\ ${ }^{2}$ Department of Pathology, Government Medical College, Patiala, \\ Punjab, India \\ ${ }^{3}$ Department of Obstetrics and Gynecology, MM Institute of \\ Medical Sciences \& Research, Mullana, Haryana, India \\ J Lab Physicians:2020;12:165-170
}

Lavleen Bharti²

\begin{abstract}
Address for correspondence Monika Garg, MD, Department of Pathology, Government Medical College, Patiala 147001, Punjab, India (e-mail: monikakash7@gmail.com).
\end{abstract}

\begin{abstract}
Background Endometrial carcinoma is often preceded by characteristic histopathologic lesions known as endometrial hyperplasia. Estrogen, p53, PTEN, and overexpression of cyclin D1 appear to be involved in the development of endometrial carcinogenesis.

Design We evaluated and compared the expression profile of cyclin D1 expressions in 50 endometrial samples submitted as either endometrial curetting $(n=34)$ or hysterectomy $(n=16)$ specimens, which were diagnosed as simple hyperplasia $(n=10)$, complex hyperplasia $(n=06)$, atypical hyperplasia $(n=04)$, and endometrial carcinoma $(n=20)$. Ten cases of normal proliferative and secretory endometrium were selected as controls. Breast cancer with known cyclin D1 expression was selected as a positive control in each immunohistochemistry run.

Results Cyclin D1 was significantly overexpressed in glands with complex hyperplasia and endometrial adenocarcinoma compared with proliferative or secretory endometrium and simple hyperplasia. A statistical difference was found in the extent of cyclin D1 positivity of simple hyperplasia and carcinoma of the endometrium $(p<0.005)$. No statistical difference was seen between complex hyperplasia and carcinoma and clinicopathologic parameters in endometrioid carcinomas. All cases of clear cell carcinoma and serous carcinoma showed cyclin D1 immunoreactivity. Significant statisti-

Keywords

- carcinoma

- cyclin D1

- endometrium

- hyperplasia

- immunohistochemistry cal difference was seen between cyclin D1 expression and only one clinicopathologic parameter, i.e., menopausal status in endometrial carcinomas

Conclusion Cyclin D1 over expression may be an early event in endometrial carcinogenesis and cyclin D1 over expression may be an informative biomarker to recognize subsets of endometrial lesions that may be precancerous and therefore amenable to surgical therapy.
\end{abstract}

Organization where the primary research was conducted: MM Institute of Medical Sciences \& Research, Mullana, Haryana, India
DOI https://doi.org/ $10.1055 / \mathrm{s}-0040-1721150$ ISSN 0974-2727. (c) 2020. The Indian Association of Laboratory Physicians.

This is an open access article published by Thieme under the terms of the Creative Commons Attribution-NonDerivative-NonCommercial-License, permitting copying and reproduction so long as the original work is given appropriate credit. Contents may not be used for commercial purposes, or adapted, remixed, transformed or built upon. (https://creativecommons.org/licenses/by-nc-nd/4.0/)

Thieme Medical and Scientific Publishers Pvt. Ltd., A-12, 2nd Floor, Sector 2, Noida-201301 UP, India 


\section{Introduction}

Endometrial carcinoma is often preceded by endometrial hyperplasia. Hyperplasia is usually associated with exogenous estrogen stimulation and thus, estrogen is considered as an endometrial carcinogen. There is a continuum of changes evolving to endometrial carcinoma. ${ }^{1}$

Many of the genes involved in cell cycle progression are frequently mutated in human cancers leading to uncontrolled cell division and tumor growth. Endometrial carcinogenesis includes a mutation in p53, PTEN tumor suppressor gene, and overexpression of cyclin D1. Mutation in p53 has been detected in serous endometrial carcinoma. Mutation/overexpression of PTEN has been reported in most of the endometrial carcinoma. Many of the genes involved in cell cycle progression are frequently mutated in human cancers leading to uncontrolled cell division and tumor growth. Cyclin D1 is a member of the cyclin G1 family and controls the transition from G1 to $S$ phase in the cell cycle. The cyclin D1 protein is encoded by the CCND1 proto-oncogene localized on chromosome 11q13. Binding of cyclin D1 to the cyclin-dependent kinases 4 and $6(\mathrm{CDK} 4 / 6)$ results in the formation of active complexes that phosphorylate the retinoblastoma tumor suppressor gene during the G1 phase. ${ }^{2}$ Mutation, amplification, and overexpression of the CCND1 gene, which can alter cell cycle progression, are observed frequently in a variety of tumors and may contribute to tumorigenesis. Multiple studies have shown cyclin D1 overexpression as a potential biomarker for precancerous and cancerous endometrial lesions, however, whether it participates in a causative or incidental manner in tumor progression is still to be determined., ${ }^{3,4}$

This study was aimed to check the relationship between cyclin D1 expression in various endometrial lesions and clinicopathological parameters to explore the possibility for cyclin D1 as a potential diagnostic marker to distinguish these endometrial lesions.

\section{Material and Methods}

This study was a prospective case-control study; 50 cases of endometrial biopsy/hysterectomy specimens were selected randomly for the study from the period of October 2013 to September 2015. Uncooperative and nonconsenting patients were excluded. The study was done after approval from the Institutional Ethical Committee. The histological diagnosis and staging were assigned based on the criteria established by the International Federation of Gynecology and Obstetrics Staging of Endometrial Cancer, 2009. ${ }^{5}$ Out of 50,16 hysterectomy specimens and 34 endometrial biopsies were included in the study. The samples included simple hyperplasia without atypia $(n=10)$, complex hyperplasia without atypia $(n=6)$, complex hyperplasia with atypia $(n=4)$, and endometrial carcinoma $(n=20)$. Ten cases of normal proliferative and secretory endometrium were selected as control. Special histochemical stains were applied as per standard techniques. Immunohistochemistry was performed using rabbit monoclonal antibody technology (clone: EP12 manufactured by DAKO Epitomics Inc.).
The streptavidin-biotin method was performed on the paraffin sections to study the expression of cyclin D1. Sections of 4 microns size were deparaffinized with xylene and rehydrated through graded alcohol. Antigen retrieval was done using Tris EDTA in the microwave. Sections were rinsed in tris-buffered saline (TBS) and then treated with FLEX peroxidase blocking reagent (DM821). Then sections were incubated with FLEX monoclonal rabbit anti-human cyclin D1 antibody (Code ISO83). The slides were washed and incubated with FLEX/HRP (Code DM822) and then washed with TBS. An avidin-biotin-peroxidase complex (DAKO, North America, United States) with DAB (3,3'-diaminobenzidine) as chromogen was used for detecting antibody binding. Slides were counterstained with Mayer's hematoxylin. Representative samples of breast carcinoma served as a positive control for cyclin D1 antibody. Cyclin D1 staining was assessed in the glandular epithelium according to the number, distribution, and intensity of the positively stained cells.

Cyclin D1 was evaluated for intensity and extent of nuclear positivity. Due to heterogeneity of the reaction, the area with the highest level of nuclear staining was selected and the number of positively stained nuclei in 1,000 glandular cells was counted under 40×magnification and results were expressed as percentage staining. Staining of cyclin D1 was evaluated according to the number, distribution, and intensity of the positively stained cells. The intensity was graded as $0=$ no staining; $1+=$ weak nuclear staining (faint chromatin pattern); $2+=$ moderate nuclear staining (chromatin clearly defined); and $3+=$ marked nuclear staining (chromatin obscured by staining) and the extent of staining was graded on a scale of 1 to 100 and scoring was done as < $10 \%=0 ; 11$ to $30 \%=1+; 31$ to $60 \%=2+$, and 60 to $100 \%=3+$.

\section{Results}

The age of the patients was in the range of 26 to 75 years. Out of 20 cases, 15 (75\%) with endometrial carcinoma were in the post-menopausal age group. In complex hyperplasia, four cases were of atypia while six cases were complex hyperplasia without atypia ( - Fig. 1). - Table 1 shows the distribution of different endometrial lesions according to age groups.

Amongst endometrial carcinoma, 15 cases (60\%) were of endometrioid type (two cases revealed villoglandular pattern while squamous morules were seen in three cases), three cases were reported to be clear cell type and two cases were papillary serous carcinoma ( $\boldsymbol{- F i g s .} \mathbf{2}$ and $\mathbf{3}$ ). Ten cases (50\%) of endometrial carcinoma were found to be in grade I, three cases (15\%) were in grade II, and seven cases (35\%) including clear cell and papillary serous carcinoma were in grade III. Nine cases of endometrial carcinoma were considered for staging as only hysterectomy specimens could be assessed. Six cases (66.7\%) were found to be in Stage IA, two cases (22.2\%) were in Stage II, and one case (11.1\%) in Stage III. Out of nine, six cases (66.67\%) showed less than half myometrial invasion and three cases (33.33\%) showed more than half myometrial invasion.

Expression of intensity and extent of cyclin D1 in endometrial diseases: - Table 2 shows the nuclear intensity and extent of 

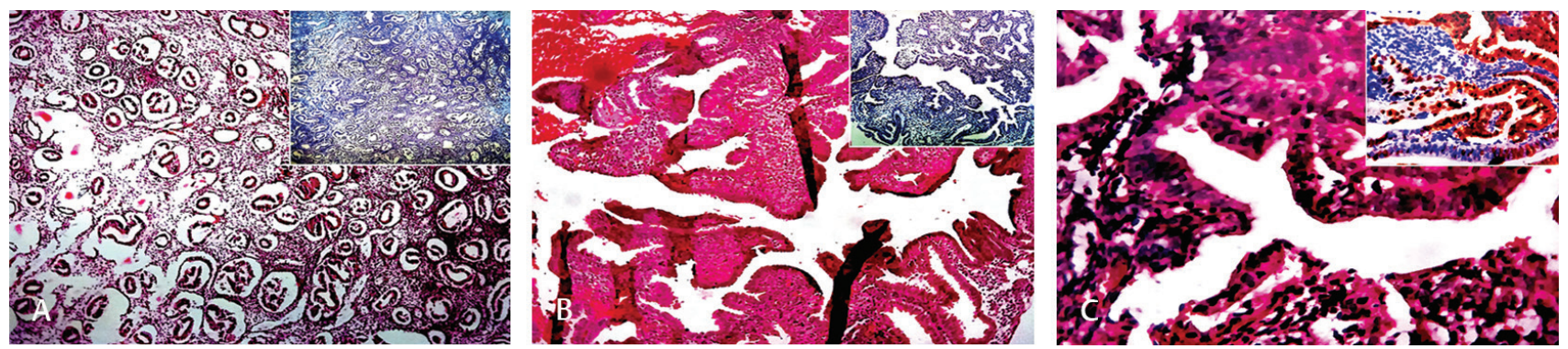

Fig. 1 (A) Low power (10x) view showing simple hyperplasia without atypia (H\&E) inset showing negative cyclin D1 expression, intensity 1+, extent $0(<5 \%)$. (B) Low power $(10 \times)$ view showing complex hyperplasia without atypia (H\&E) inset showing negative cyclin D1 expression, intensity $1+$, extent $0(5-10 \%)$. (C) High power (40x) view showing simple hyperplasia with atypia (H\&E) inset showing positive cyclin D1 expression, intensity $2+$, extent 3+ (70-80\%). H\&E, hematoxylin and eosin.

Table 1 Distribution of various endometrial lesions according to age $(n=50)$

\begin{tabular}{|c|c|c|c|c|c|c|c|c|}
\hline \multirow[t]{2}{*}{$\begin{array}{l}\text { Age group } \\
\text { (y) }\end{array}$} & \multicolumn{2}{|c|}{ No. of cases } & \multicolumn{2}{|c|}{$\begin{array}{l}\text { Simple hyperplasia } \\
\qquad(n=10)\end{array}$} & \multicolumn{2}{|c|}{$\begin{array}{l}\text { Complex hyperplasia } \\
\qquad(n=10)\end{array}$} & \multicolumn{2}{|c|}{$\begin{array}{l}\text { Malignant endometrial tumor } \\
\qquad(n=20)\end{array}$} \\
\hline & $N$ & $\%$ & $N$ & $\%$ & $N$ & $\%$ & $N$ & $\%$ \\
\hline $21-30$ & 01 & $2 \%$ & - & - & - & - & - & - \\
\hline $31-40$ & 16 & $32 \%$ & 3 & $30 \%$ & 5 & $50 \%$ & 2 & $10 \%$ \\
\hline $41-50$ & 15 & $30 \%$ & 6 & $60 \%$ & 2 & $20 \%$ & 4 & $20 \%$ \\
\hline $51-60$ & 13 & $26 \%$ & 1 & $10 \%$ & 3 & $30 \%$ & 9 & $45 \%$ \\
\hline $61-70$ & 04 & $8 \%$ & - & - & - & - & 4 & $20 \%$ \\
\hline$>70$ & 01 & $2 \%$ & - & - & - & - & 1 & $5 \%$ \\
\hline Mean age & \multicolumn{2}{|c|}{46.1} & \multicolumn{2}{|c|}{42.0} & \multicolumn{2}{|c|}{43.0} & \multicolumn{2}{|c|}{54.25} \\
\hline
\end{tabular}
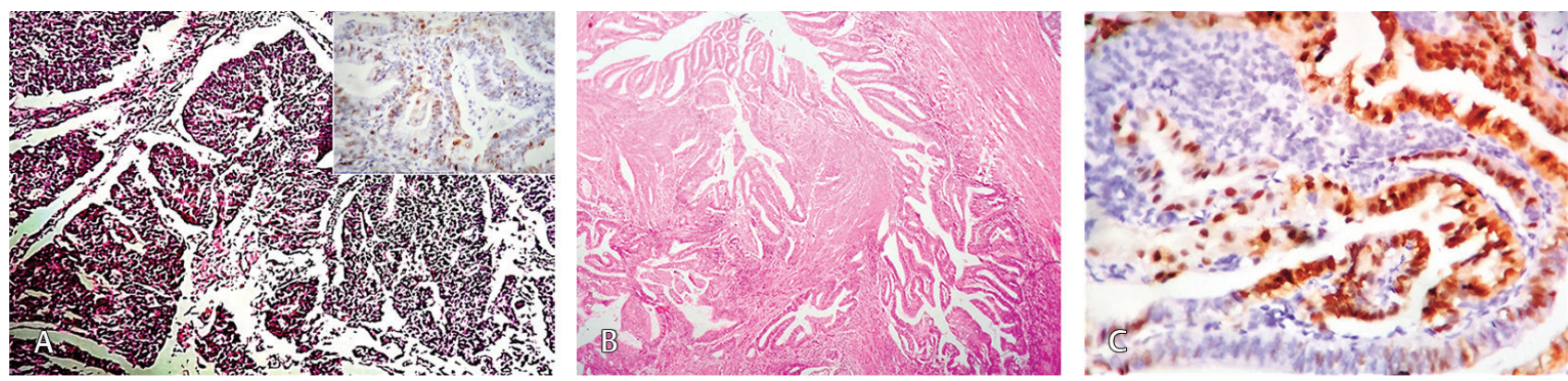

Fig. 2 (A) Low power (10×) view showing endometrioid carcinoma in a glandular pattern (H\&E) inset showing positive cyclin D1 expression, intensity $2+$, extent $1+(10-15 \%)$. (B) Low power (10×) view showing endometrioid carcinoma villoglandular type (H\&E). (C) Low power (10×) view showing endometrioid carcinoma villoglandular type (H\&E) positive cyclin D1 expression, intensity 3+, extent 2+ (50\%). H\&E, hematoxylin and eosin.
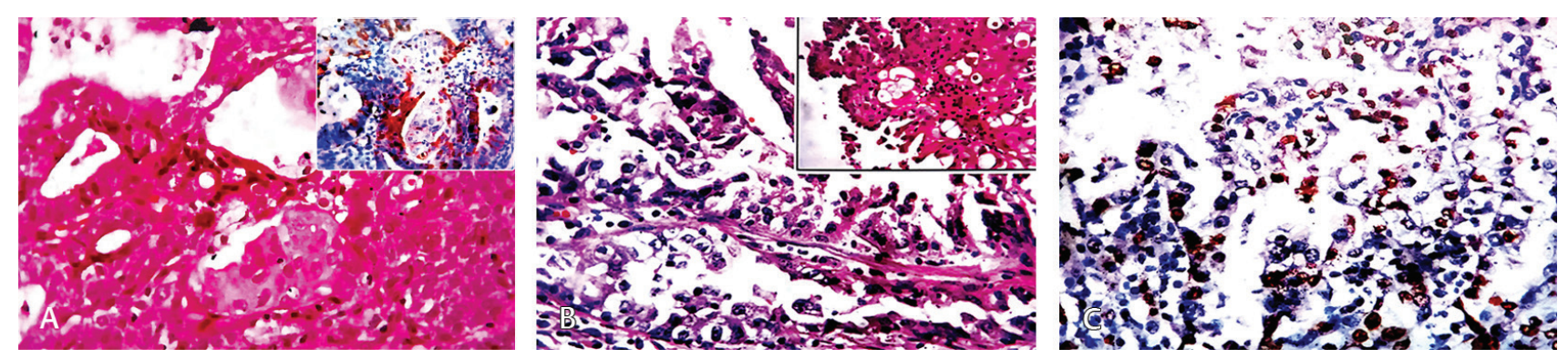

Fig. 3 (A) High power (40x) view showing endometrioid carcinoma arranged in glands with squamous morules (adenoacanthoma) (H\&E) inset showing positive cyclin D1 expression, intensity 2+, extent 1+ (20\%). (B) High power (40×) view showing clear cell carcinoma of endometrium, a cell having abundant clear cytoplasm with high-grade nuclei and hob-nailing, inset showing prominent hob-nail appearance (H\&E). (C) High power $(40 \times)$ view showing clear cell carcinoma of endometrium, positive for cycleinD1 expression, intensity $2+$, extent $2+(30-40 \%)$. H\&E, hematoxylin and eosin. 
Table 2 Immunoreactivity of cyclin D1 (extent and intensity) in normal and various endometrial lesions

\begin{tabular}{|c|c|c|c|c|c|c|c|c|c|c|c|c|c|c|c|c|c|c|}
\hline \multirow[t]{3}{*}{ Lesion } & \multirow{2}{*}{\multicolumn{2}{|c|}{$\begin{array}{c}\text { No. } \\
\text { of cases }\end{array}$}} & \multicolumn{8}{|c|}{ Extent } & \multicolumn{8}{|c|}{ Intensity } \\
\hline & & & \multicolumn{2}{|r|}{0} & \multicolumn{2}{|r|}{1} & \multicolumn{2}{|c|}{2} & \multicolumn{2}{|r|}{3} & \multicolumn{2}{|r|}{0} & \multicolumn{2}{|r|}{1} & \multicolumn{2}{|c|}{2} & \multicolumn{2}{|r|}{3} \\
\hline & $N$ & $\%$ & $N$ & $\%$ & $N$ & $\%$ & $N$ & $\%$ & $N$ & $\%$ & $N$ & $\%$ & $N$ & $\%$ & $N$ & $\%$ & $N$ & $\%$ \\
\hline $\begin{array}{l}\text { Proliferative } \\
\text { endometrium }\end{array}$ & 05 & $10 \%$ & - & - & - & - & - & - & - & - & - & - & - & - & - & - & - & - \\
\hline $\begin{array}{l}\text { Secretory } \\
\text { endometrium }\end{array}$ & 05 & $10 \%$ & - & - & - & - & - & - & - & - & - & - & - & - & - & - & - & - \\
\hline $\begin{array}{l}\text { Simple } \\
\text { hyperplasia }\end{array}$ & 10 & $20 \%$ & 7 & $70 \%$ & 3 & $30 \%$ & - & - & - & - & 3 & $30 \%$ & 7 & $70 \%$ & - & - & - & - \\
\hline $\begin{array}{l}\text { Complex } \\
\text { hyperplasia }\end{array}$ & 10 & $20 \%$ & 2 & $20 \%$ & 3 & $30 \%$ & 4 & $40 \%$ & 1 & $10 \%$ & - & - & 5 & $50 \%$ & 5 & $50 \%$ & - & - \\
\hline $\begin{array}{l}\text { Endometrial } \\
\text { carcinoma }\end{array}$ & 20 & $40 \%$ & 4 & $20 \%$ & 4 & $20 \%$ & 11 & $55 \%$ & 1 & $5 \%$ & - & - & 6 & $30 \%$ & 10 & $50 \%$ & 4 & $20 \%$ \\
\hline
\end{tabular}

Table 3 Comparison of extent and intensity of cyclin D1 expression

\begin{tabular}{|c|c|c|c|c|c|c|c|}
\hline \multicolumn{2}{|c|}{ Parameter $a$ vs. parameter $b$} & \multicolumn{2}{|c|}{$\begin{array}{l}\text { Positives } \\
\text { (Parameter } a \text { ) }\end{array}$} & \multicolumn{2}{|c|}{$\begin{array}{r}\text { Positives } \\
\text { (Parameter } b)\end{array}$} & \multirow[t]{2}{*}{$\chi^{2}$} & \multirow[t]{2}{*}{$p$-Value } \\
\hline & & $N$ & $\%$ & $N$ & $\%$ & & \\
\hline \multirow{2}{*}{$\begin{array}{l}\text { Simple hyperplasia vs. } \\
\text { complex hyperplasia }\end{array}$} & Extent & $3 / 10$ & $30 \%$ & $8 / 10$ & $80 \%$ & 5.051 & 0.070 NS \\
\hline & Intensity & $7 / 10$ & $70 \%$ & $10 / 10$ & $100 \%$ & 3.529 & $0.211 \mathrm{NS}$ \\
\hline \multirow{2}{*}{$\begin{array}{l}\text { Simple hyperplasia vs. } \\
\text { carcinoma }\end{array}$} & Extent & $3 / 10$ & $30 \%$ & $16 / 20$ & $80 \%$ & 7.177 & $0.015 \mathrm{~S}$ \\
\hline & Intensity & $7 / 10$ & $70 \%$ & $20 / 20$ & $100 \%$ & 6.667 & $0.030 \mathrm{~S}$ \\
\hline \multirow{2}{*}{$\begin{array}{l}\text { Complex hyperplasia vs. } \\
\text { carcinoma }\end{array}$} & Extent & $8 / 10$ & $80 \%$ & $16 / 20$ & $80 \%$ & 0 & $1.000 \mathrm{NS}$ \\
\hline & Intensity & $10 / 10$ & $100 \%$ & $20 / 20$ & $100 \%$ & NA & NA \\
\hline
\end{tabular}

Abbreviation: NA, not applicable; NS, not specified.

immuno-expression of cyclin D1 in normal proliferative, hyperplastic, and neoplastic endometrial samples. It was observed that all the cases of clear cell carcinoma and serous carcinoma gave positivity for cyclin D1 while 11 out of 15 (73.33\%) endometrioid carcinoma were positive for cyclin D1.

Statistical comparison of cyclin D1 expression in endometrial diseases: Statistical analysis showed a significant difference in extent as well as the intensity of cyclin D1 immunoreactivity between simple hyperplasia and carcinoma ( $p=0.015$ and $p=0.030$, respectively). No significant difference was found between simple hyperplasia and complex hyperplasia ( - Table 3 ).

Correlation of cyclin D1 with various clinicopathological features: Various clinicopathological parameters were correlated with cyclin D1 expression. Statistical analysis showed a significant difference with menopausal status $(p=0.033)$. No significant correlation was found with other parameters including myometrial invasion, grade, and stage of the tumor.

\section{Discussion}

Endometrial cancer is the fourth most common cancer occurring in women after breast, bowel, and lung cancers and is derived from the endometrial epithelial lining of the uterine corpus. ${ }^{6}$ Endometrial carcinoma is often preceded by endometrial hyperplasia. Hyperplasia is usually associated with exogenous estrogen stimulation and thus, estrogen is considered as an endometrial carcinogen. There is a continuum of changes evolving to endometrial carcinoma. ${ }^{1}$ Clinicopathological, immunohistochemical, and molecular genetic studies during the last two decades have generated substantial data to support the development of endometrial carcinoma. ${ }^{7,8}$

Immunohistochemical methods have been useful for detecting several biomarkers of possible prognostic importance for several cancer types. Cyclin D1 is a key protein in the regulation of the cell cycle at the $\mathrm{G} 1$ to $\mathrm{S}$ phase transition and is essential for the regulation of proliferation, differentiation, and transcriptional control. Overexpression of cyclin D1 induces excessive cellular proliferation and is often overexpressed in human neoplasias, e.g., in situ and infiltrating ductal breast carcinoma, colorectal carcinoma, bladder carcinoma, head and neck, lung and prostate cancers by rearrangement, amplification, and mutation of the genes. ${ }^{9}$

Overexpression of cyclin D1 in endometrial cancer has been reported in previous studies but very few studies have compared the expression of cyclin D1 in various endometrial conditions. In the present study, none of the cases of proliferative and secretory endometrium showed positivity for cyclin D1. Three out of ten cases (30\%) of simple hyperplasia were positive for cyclin D1. In complex hyperplasia, eight out of ten cases (80\%) gave positivity for cyclin D1. All these findings correspond to studies done by Ruhul Quddus et al, ${ }^{10}$ Özuysal et al, ${ }^{11}$ Choudhary and Bansal, ${ }^{12}$ Liang et al,,${ }^{13}$ Stewart et al, ${ }^{14}$ and Shevra et al. ${ }^{15}$ Increased expression by cyclin D1 was seen in few cases of simple hyperplasia and complex hyperplasia 
without atypia. The reason could be explained by the fact that as the normal proliferative cell enters the cell cycle from $G_{0}$ phase, cyclin D1 is localized in the nucleus early in the $\mathrm{G}_{1}$ phase, and exits as the cell progresses into the $\mathrm{S}$ phase. One case of complex hyperplasia with atypia showed foci of extensively crowded glands showing atypia with cyclin D1 expression in almost 70\% of nuclei, strongly suggesting that the lesion is potentially premalignant.

In endometrial carcinoma, out of 20 cases, 16 cases gave cyclin D1 positivity. Four cases (20\%) showed an extent of 1+, 11 cases (55\%) showed an extent of $2+$, and one case (05\%) showed an extent of $3+$. Shawana et al revealed strong staining in $>30 \%$ of nuclei in eight $(44.4 \%)$ cases of endometrial adenocarcinoma. $^{3}$

Consistent with previous findings, we observed a gradual and progressive increase in expression of cyclin D1 levels in normal proliferative, hyperplastic endometrium, atypical hyperplastic proliferation, and endometrial carcinoma. - Table 4 shows a comparison of cyclin D1 reactivity in simple, complex hyperplasia, and endometrial carcinoma. The mechanism of cyclin D1 dysregulation is likely to contribute to an increase in the proportion of cells in the transition from $G_{1}$ to $S$ phase.

Statistical analysis of the extent of cyclin D1 immunoreacti vity showed that in simple hyperplasia and carcinoma endometrium, the difference was statistically significant $(p=$ 0.015 ) while in simple hyperplasia versus complex hyperplasia and complex hyperplasia versus carcinoma, the difference was not statistically significant. In our study, no statistical correlation was found in simple hyperplasia versus complex hyperplasia and complex hyperplasia versus carcinoma while in other studies there was a significant statistical difference. ${ }^{10}$ This discordance may be due to the small sample size in our study.

Ruhul Quddus et al analyzed immunoreactivity in five groups (proliferative, secretary, simple hyperplasia, complex hyperplasia with or without atypia, and endometrial carcinoma) and have shown that they are statistically different groups. Their observations were similar to ours and have documented no difference between complex hyperplasia and carcinoma and between proliferative, secretory, and simple hyperplasia. They did not segregate atypical hyperplasia as a separate category. Instead, they merged it with the complex hyperplasia category in their study. Significant overexpression was also noted in papillary, syncytial, and squamous metaplasia compared with normal surface epithelium or epithelium with tubal metaplasia. They suggested that maximal dysregulation occurs at the complex hyperplasia state and concluded that some alterations might be responsible for the different morphologic features and behavior of complex hyperplasia and carcinoma. Authors have also suggested that cyclin D1 overexpression might be an early event in endometrial carcinogenesis. ${ }^{10}$

Liang et al checked the relationship between its expression and clinicopathological variables of endometrial lesions to explore the possibility for cyclin D1 as a potential diagnostic and prognostic marker. The results of this research showed the expression of cyclin D1 increased in the order of simple hyperplasia, complex hyperplasia, endometrioid carcinoma, clear cell carcinoma except for endometrial serous carcinoma; the statistical comparisons of these types with each other demonstrated no statistical significance $(p>0.005) .{ }^{13}$

Shevra et al evaluated and compared the expression profile of cyclin D1 and $\mathrm{K}_{\mathrm{i}}-67$ expressions in 61 endometrial samples. There was increased expression of cyclin D1 and $\mathrm{K}_{\mathrm{i}}-67$ in patients with endometrial carcinoma relative to proliferative endometrium and simple hyperplasia, but there was no such difference between cases of atypical hyperplasia and endometrial carcinoma. ${ }^{15}$

Kala et al evaluated the expression of cyclin D1 in hyperplasia and endometrial carcinoma and compared the variation of cyclin D1 expression in various tumor types, especially serous and endometrioid carcinomas. A statistical difference was found in the extent of cyclin D1 positivity of complex hyperplasia and carcinoma of the endometrium. None of the cases of serous carcinoma showed cyclin D1 immunoreactivity. No correlation was seen between cyclin D1 expression and clinicopathologic parameters in endometrioid carcinomas. It was inferred that cyclin D1 expression is a quantitative molecular dysregulation that is increasing progressively from complex hyperplasia to carcinoma of the endometrium. ${ }^{16}$

Various clinicopathological parameters were correlated with cyclin D1 expression. Contrary to various studies our findings revealed significant statistical difference with menopausal status $(p=0.033){ }^{13,17}$ No significant correlation was found with other parameters including grade, stage, and myometrial invasion of the tumor similar to study done by Liang et al and Kala et al. ${ }^{13,16,18}$

\section{Conclusion}

Our findings suggest that cyclin D1 is dysregulated and therefore may have a role in endometrial carcinogenesis.

Table 4 Comparison of cyclin D1 immunoreactivity

\begin{tabular}{|l|l|l|l|l|}
\hline \multirow{2}{*}{ Study } & \multirow{2}{*}{ Year } & \multicolumn{3}{|c|}{ Cyclin D1 expression in } \\
\cline { 3 - 5 } & & Simple hyperplasia & Complex hyperplasia & Carcinoma endometrium \\
\hline Ruhul Quddus et al $^{10}$ & 2002 & $57 \%$ & $71 \%$ & $68 \%$ \\
\hline Özuysal et al $^{11}$ & 2005 & $0 \%$ & $1 \%$ & $26.6 \%$ \\
\hline Choudhary et al $^{12}$ & 2006 & $0 \%$ & $33 \%$ & $50 \%$ \\
\hline Liang et al $^{13}$ & 2013 & $30 \%$ & $49 \%$ & $67 \%$ \\
\hline Our study & 2015 & $30 \%$ & $80 \%$ & $80 \%$ \\
\hline
\end{tabular}


Since there was a statistically significant difference in cyclin D1 expression between simple hyperplasia and carcinoma, it appears that the dysregulation is maximal at the level of complex hyperplasia and that cyclin D1 overexpression may be an early event in endometrial carcinogenesis. Our findings support the significance of complex hyperplasia as a precursor lesion and to some extent, simple hyperplasia is also precancerous. Thus, cyclin D1 overexpression may be an informative biomarker to recognize subsets of endometrial lesions that may be precancerous and therefore amenable to surgical therapy.

\section{Conflict of Interest}

None declared.

\section{References}

1 Silverberg SG, Kurman RJ, Tumors of the uterine corpus and gestational trophoblastic disease. Atlas of Tumor Pathology: Third series, Fascicle 3. Washington, DC: Armed Forces Institute of Pathology; 199:2 290

2 Wang Y, Zhu JF, Liu YY, Han GP. An analysis of cyclin D1, cytokeratin 5/6 and cytokeratin 8/18 expression in breast papillomas and papillary carcinomas. Diagn Pathol 2013;8:8

3 Shawana S, Kehar SI, Masood S, Aamir I. Immunoexpression of cyclin D1 and PTEN in various endometrial pathologies. J Coll Physicians Surg Pak 2016;26(4):277-282

$4 \mathrm{Wu} \mathrm{W}$, Slomovitz BM, Soliman PT, et al. Correlation of cyclin D1 and cyclin D3 overexpression with the loss of PTEN expression in endometrial carcinoma. Int J Gynecol Cancer 2006;16(4):1668-1672

5 Abu-Rustum NR, Zhou $Q$ Iasonos $A$, et al. The revised 2009 FIGO staging system for endometrial cancer: should the 1988 FIGO stages IA and IB be altered? Int J Gynecol Cancer 2011;21(3):511-516

6 Gayther SA, Pharoah PD. The inherited genetics of ovarian and endometrial cancer. Curr Opin Genet Dev 2010;20(3):231-238

7 Bokhman JV. Two pathogenetic types of endometrial carcinoma. Gynecol Oncol 1983;15(1):10-17
8 Sun Y, Luo D, Liao DJ. Cyclin D1 protein plays different roles in modulating chemoresponses in MCF7 and MDA-MB231 cells. J Carcinog 2012;11(1):12

9 Sherr CJ, Roberts JM. CDK inhibitors: positive and negative regulators of G1-phase progression. Genes Dev 1999; 13(12):1501-1512

10 Ruhul Quddus M, Latkovich P, Castellani WJ, et al. Expression of cyclin D1 in normal, metaplastic, hyperplastic endometrium and endometrioid carcinoma suggests a role in endometrial carcinogenesis. Arch Pathol Lab Med 2002;126(4):459-463

11 Özuysal S, Oztürk H, Bilgin T, Filiz G. Expression of cyclin D1 in normal, hyperplastic and neoplastic endometrium and its correlation with Ki-67 and clinicopathological variables. Arch Gynecol Obstet 2005;271(2):123-126

12 Choudhury M, Bansal S. Expression of cyclin D1 in endometrial hyperplasia and endometrial carcinoma. Indian J Pathol Microbiol 2007;50(4):708-710

13 Liang S, Mu K, Wang Y, et al. CyclinD1, a prominent prognostic marker for endometrial diseases. Diagn Pathol 2013;8:138

14 Stewart CJR, Crook ML, Leung YC, Platten M. Expression of cell cycle regulatory proteins in endometrial adenocarcinoma: variations in conventional tumor areas and in microcystic, elongated and fragmented glands. Mod Pathol 2009;22(5):725-733

15 Shevra CR, Ghosh A, Kumar M. Cyclin D1 and Ki-67 expression in normal, hyperplastic and neoplastic endometrium. J Postgrad Med 2015;61(1):15-20

16 Kala M, Gill M, Gupta V, Srivastava D, Tanwar P, Goyal V. Cyclin D1 expression in hyperplasia and carcinoma of the endometrium and its correlation with histologic grade and tumor type. J Gynecol Surg 2014;30(1):1-4

17 Nishimura Y, Watanabe J, Jobo T, et al. Cyclin D1 expression in endometrioid-type endometrial adenocarcinoma is correlated with histological grade and proliferative activity, but not with prognosis. Anticancer Res 2004;24(4):2185-2191

18 Khabaz MN, Abdelrahman AS, Butt NS, Al-Maghrabi B, Al-Maghrabi J. Cyclin D1 is significantly associated with stage of tumor and predicts poor survival in endometrial carcinoma patients. Ann Diagn Pathol 2017;30:47-51 